\title{
AUтновs Real-time Load Determination of Wheel Loader Components
}

Lars Brinkschulte,

M. Sc.

is Research Assistant at the Institute of Vehicle

System Technology of the

Karlsruhe Institute of

Technology (KIT) in

Karlsruhe (Germany).
Adapted vehicle control systems can reduce the stresses and damage, the machine downtime and consequently the operating and maintenance costs of mobile machines. Researchers of the Karlsruhe Institute of Technology (KIT) have therefore developed a method for real-time load determination using the example of a wheel loader boom.
Julian Hafner, M. Sc. is a former student work at the Institute of Vehicle System Technology, and graduate of the Karlsruhe Institute of Technology (KIT) in Karlsruhe (Germany).

Prof. Dr.-Ing. Marcus Geimer

is Director of the Institute of Vehicle System Technology of the Karlsruhe Institute of Technology (KIT) in Karlsruhe (Germany).

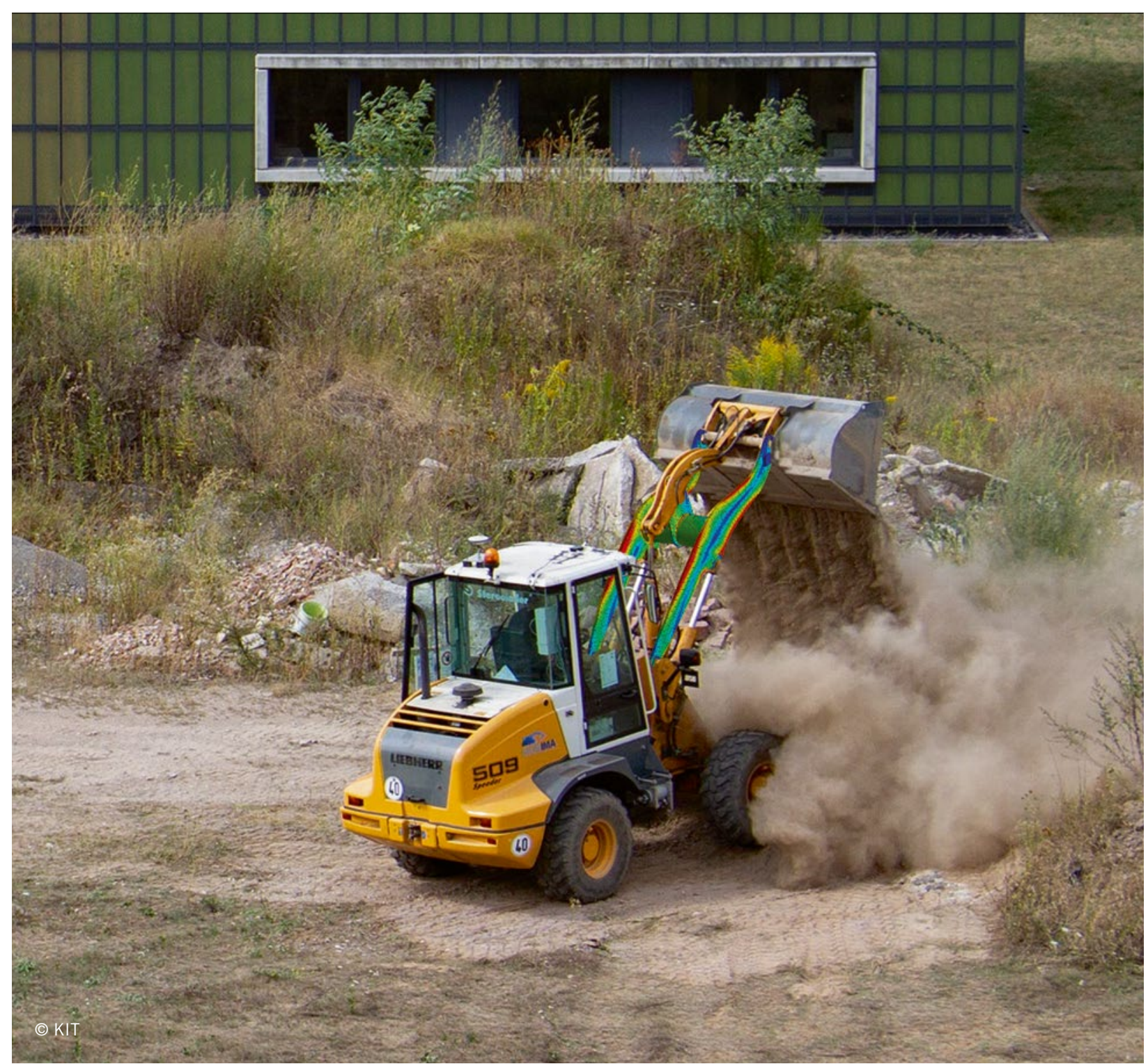


1 INTRODUCTION AND BACKGROUND

2 METHOD OF REAL-TIME LOAD ASSESSMENT

FUNDAMENTAL KINEMATIC RELATIONS

DETERMINATION OF PAYLOAD AND FORCES

5 STRESS CALCULATION

6 SUMMARY AND CONCLUSION

\section{INTRODUCTION AND BACKGROUND}

The lifecycle costs of systems are moving more and more into the focus of machine manufacturers to ensure competitive edges and strengthen customer relations. This results in development goals such as higher availability, maintenance intervals according to requirements, and minimized outages and downtime costs.

Mobile machines are subjected to loads which, occasionally, are characterized by the working task, the environmental boundary conditions and the operator. In practical use of mobile machines, some operators are not very experienced in handling a machine, for instance when using machines in the construction industry. In these cases, performing the working task enjoys priority over the state of a machine and its service life. The situation is aggravated by the fact that entrepreneurs - especially in the fields of add-on and special machines - prefer to use leased vehicles. Thus, operators are confronted with new machines all the time. Because of the individual control characteristics of operators, this results in permanently changing sequences of motions and load situations for the machine. The high loads occur frequently in operation and can lead to premature failure of a machine. As a result, on the one hand, it will be impossible to continue the activities needed to fulfil the working task and, on the other hand, it may be necessary to repair or even scrap a machine and buy a new one. Both consequences result in economic harm for the machine owner.

In many cases, it is difficult to influence the working task and the environmental conditions established by the environment in the light of the objective to achieve the required result while simultaneously minimizing component load. In contrast, operation of a vehicle offers a more evident degree of freedom in minimizing damage. Vehicle control systems adapted to external conditions and the working task make operators and vehicles perform under operating conditions less prone to result in damage. Thus they can reduce loads and defects and, consequently, also reduce operating and maintenance costs. The final consequence is a higher availability of the machines.

A control system of this kind requires knowledge of current loads and load-bearing capabilities of components of a mobile machine. Developing a method of real-time load determination as shown for the boom of a wheel loader therefore is the content of this article.

\section{METHOD OF REAL-TIME LOAD DETERMINATION}

This method is demonstrated using the boom of a wheel loader with stereo steering, an operating weight of $5.6 \mathrm{t}$ and a maximum payload of $2 \mathrm{t}$ as an example. The selection of the load estimation of the boom is based on the increased number of failures due to false loading and overloading of such machines, as scientifically investigated in [1]. The machine is designed with a Z-kinematic, with a hydraulically pilot-controlled open-center constant-flow system.

The machine is equipped with measuring technique for various research purposes. The sensors needed to determine the load of the boom are shown in FIGURE 1. Sensors 1 to 4 are pressure sensors recording cylinder chamber pressures of the lifting and tilting cylinders with a measurement accuracy of \pm 2 bar. Sensors 5 and 6 are distance measuring instruments to record the change in cylinder stroke length with a measuring accuracy of $\pm 15 \mathrm{~mm}$.

To calculate the load, a relationship between the sensor signals and the component load must be determined. In this case, stresses at the highly loaded points of the component serve as load indicators, FIGURE 2 . The basic method is a three-step process.

Step 1 (Part 3 of this article) shows the fundamental kinematic relations of the lifting kinematics subassembly. Based on analytical approaches, the measured values of the cylinder stroke $\left(\vec{L}_{\text {cyl }}\right)$ are converted into angular relations $(\vec{\Upsilon})$ of the components moving relative to one another. Step 2 (Part 4) contains the determination of the forces acting on the component. In a first step, the bulk weight in the bucket (payload) is determined based on the cylinder pressures $\vec{p}_{\text {cyl }}$ and the angular relations $\vec{r}$. This is followed by the calculation of all acting forces $\vec{F}$ in terms of magnitude and direction, resulting from movement and payload. Step 3 (Part 5) concludes the calculation of stresses at the most critical positions of the component. The basic principles of fatigue or fracture mechanics allow determining damage to the component for recent operating cycles.

The parametrization and validation of the respective algorithms has to be carried out in advance and is achieved by various simulation techniques, as Multibody Simulation (MBS) and Finite Element Method (FEM), and supplementary measurements of a test specimen. The subsystems of the method are detailed below.

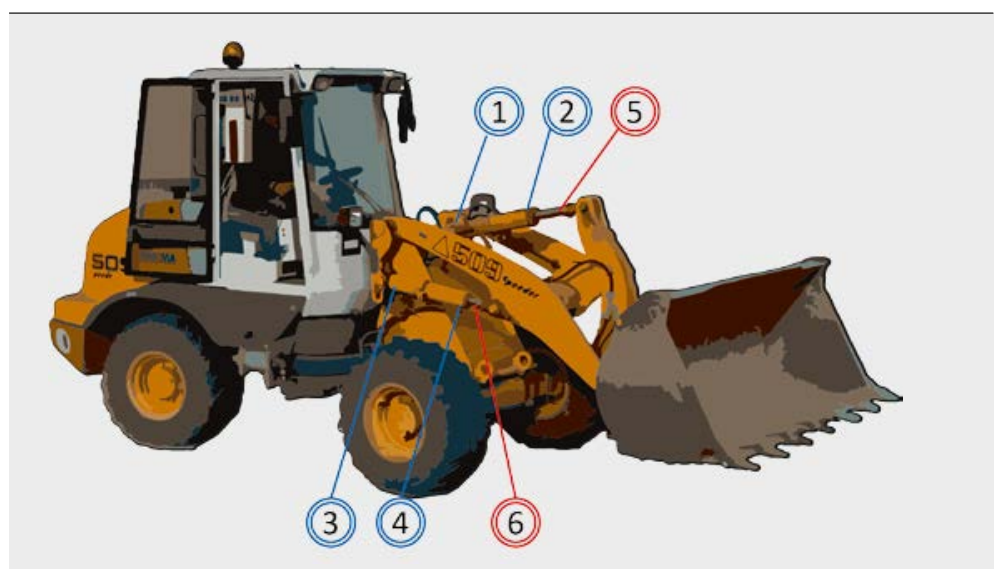

FIGURE 1 Measurement technique in machine (C KIT) 


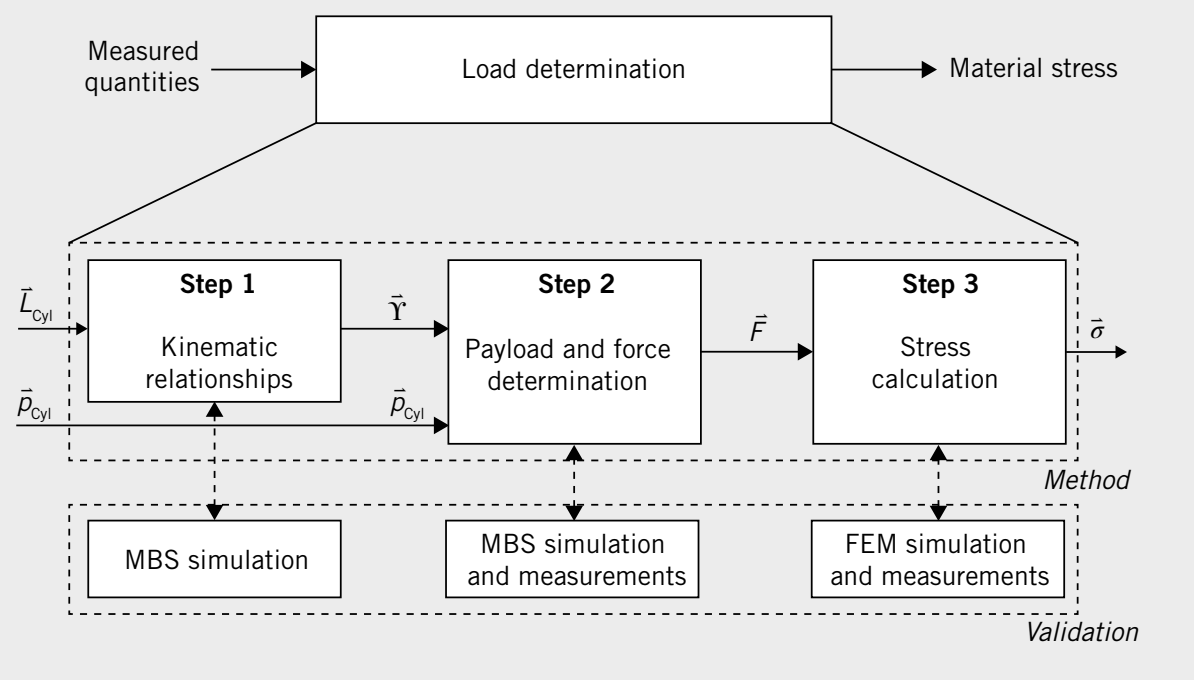

FIGURE 2 Load determination workflow (๑ KIT)

\section{FUNDAMENTAL KINEMATIC RELATIONS}

For stress calculation purposes by means of current loads, it is necessary to know the position of the lifting structure at any point in time. The fact, that the lifting kinematics is a rigid-body system with only two independent degrees of freedom, is used to determine angles and distances. In this way, the position of the lifting kinematics can be determined unambiguously by measuring two state variables. For this purpose, measurement of the cylinder stroke, which is easy to perform in practice, is used to determine the stroke length of the lifting and the tilting cylinders. The nine angles shown in FIGURE 3 are non-linearly dependent on the lengths.

As an example, the tilting angle of the bucket $\omega$ and the angle of the bar relative to the horizontal $\beta$ are demonstrated. Establishing one equation each in the $x$ - and the $y$-direction after simplification results in the relation according to Eq. 1:

$$
\begin{aligned}
& \text { Eq. } 1 \\
& -L_{\mathrm{St}}{ }^{2}+\left(\begin{array}{l}
\left(\begin{array}{l}
\sin (\omega) \cdot L_{\mathrm{Ad}}+\cos (\zeta) \cdot L_{U}-\sin \left(\varphi_{\text {mod }}\right) \cdot \\
L_{\text {mod }}+L_{\text {comb }} \cdot \sin \left(\varphi_{\text {comb }}\right)
\end{array}\right)^{2} \\
+\left(\begin{array}{l}
r_{\text {comb }} \cdot \cos \left(\varphi_{\text {comb }}\right)-\cos \left(\varphi_{\text {mod }}\right) \cdot L_{\text {mod }} \\
-\sin (\zeta) \cdot L_{\text {Div }}-\cos (\omega) \cdot L_{\text {ad }}
\end{array}\right)^{2}
\end{array}\right)=0
\end{aligned}
$$

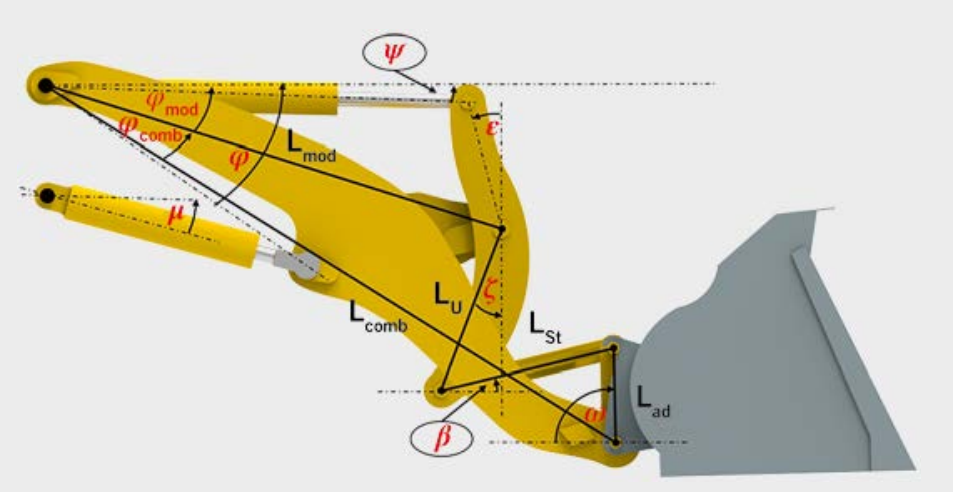

FIGURE 3 Boom kinematics (C KIT)
This equation can be used to describe the angle $\omega$ as a function of the cylinder lengths $\left(L_{\text {stroke }}\right.$ and $L_{\text {Tilt }}$ ). In this case, it applies that $\zeta=f^{\prime}\left(L_{\text {Stroke }}, L_{\text {TIlt }}\right)$ and $\varphi=f\left(L_{\text {Stroke }}, L_{\text {Tilt }}\right)$ so that consequently $\omega=f\left(L_{\text {Stroke }}, L_{\text {Tilt }}\right)$.

The other dimensions occurring are constant and can be determined from the geometry data. The non-linear solution technique Trust Region [2] is used to solve the equation.

Due to the high demand of computing capacities for a non-linear calculation, the angles are determined in advance over the entire stroke and tilt range. In real operation, the angles for the current cylinder stroke are approximated by linear interpolation between the values determined in advance. This simplified model was validated by imaging the complete kinematics in commercial MKS software tools.

\section{DETERMINATION OF PAYLOAD AND FORCES}

The stress calculation presented in Step 3 (Part 5) is based on the forces acting on the boom. The weight forces of components as well as those acting on the hydraulic cylinders - resulting from the pressures of the cylinder chambers - are known. Also the positions of the weight forces are known completely from the angles determined in Step 1 (Part 3).

The forces acting on the boom at the bearing positions in addition show significant correlation with the payload. For the calculation of the payload in a static case, two moment balances with two variables each are used. For the first balance, initially the bucket with the adapter is separated from the bar and the boom, and moment equilibrium is established around the link (blue point) to the boom, FIGURE 4. Unknown quantities of this balance are the mass force $F_{L}$ and the associated lever arm $x$, Eq. 2 .

$$
\begin{aligned}
& \text { Eq. } 2 \\
& F_{\mathrm{L}} \cdot x=\mathrm{L}_{\mathrm{Ad}} \cdot \sin (\omega+\beta) \cdot F_{\mathrm{Ad}}-F_{\mathrm{G}, \mathrm{Ad}} \cdot x_{\mathrm{Ad}}-F_{\mathrm{G}, \mathrm{Sc}} \cdot x_{\mathrm{Sc}}+F_{\mathrm{G}, \mathrm{St}} \cdot x_{\mathrm{St}}
\end{aligned}
$$

The force $F_{\text {Ad }}$ acting from the bar on the bucket can be determined by the means of the pressures acting on the tilting cylinder, the 


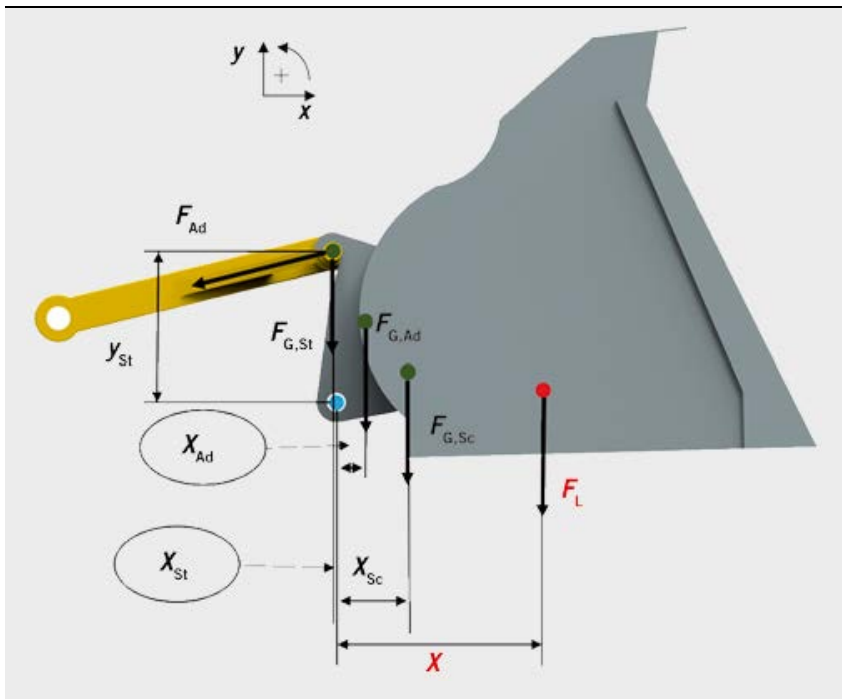

FIGURE 4 Forces acting on bucket (C KIT)

resultant cylinder force, and the lever principle acting at the force deflector.

The lifting kinematics seen as whole serves to establish the second moment balance around the bearing point of the boom, relative to the front part of the machine, FIGURE 5. The combination of the two balances allows an unequivocal calculation of the loading force of bulk material in the bucket and thus of the payload, Eq. 3 .

Eq. 3

$F_{\mathrm{L}}=\frac{1}{\mathrm{~L}_{\text {comb }} \cdot \cos \left(\varphi_{\text {comb }}\right)} \cdot\left(\begin{array}{l}-H \cdot \cos (\psi) \cdot F_{\text {TIIt }}+B \cdot \sin (\psi) \cdot F_{\text {TIIt }} \\ +F_{\text {Stroke }} \cdot r \cdot \sin (\mu-\varphi)-G-F_{L} \cdot x\end{array}\right)$

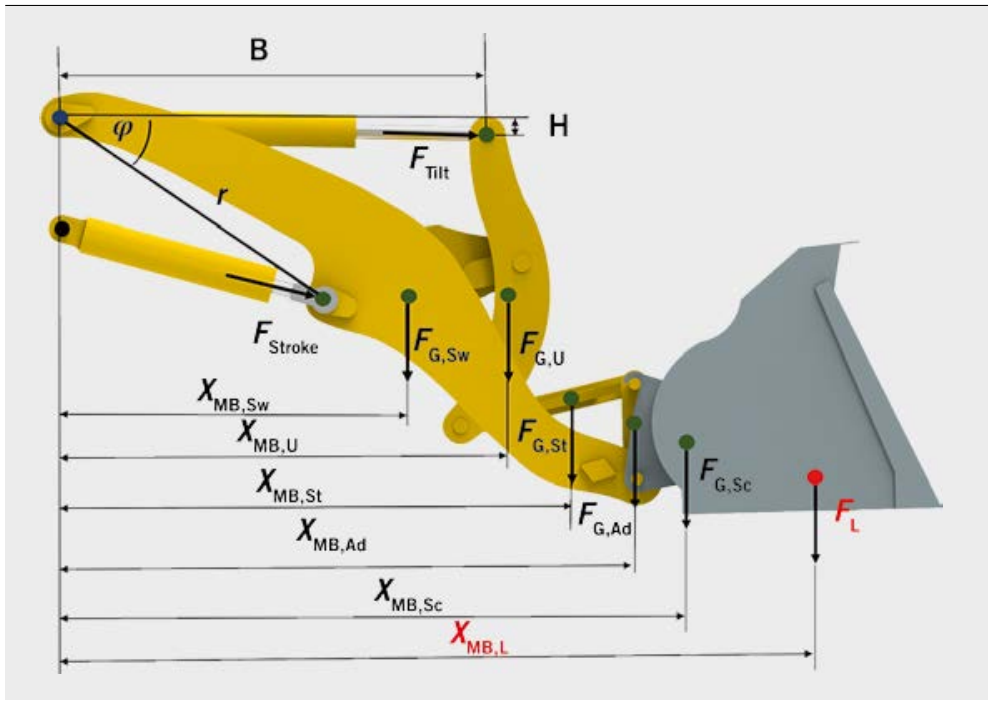

FIGURE 5 Forces acting on lifting kinematics (C KIT)

For the following determination of the component load, validating the loading force due to the payload is a factor of great importance. For this purpose, extensive series of measurements were carried out using the real machine. Each one incorporates measurements of up to 30 changing positions of the lifting and tilting cylinders at constant payload. Divergent environmental conditions, such as varying outside temperatures and bulk materials, serve to test the robustness of the algorithm. FIGURE 6 shows the results for validating the payload determination over four series of measurements spread over the entire spectrum of payloads. Deviations are presented as Full-scale (FS) deviations $e_{m, F s}$, that is the absolute error between calculation and measurements $\left(m_{\text {calc }}-m_{\text {real }}\right)$ divided by the maximum payload $\left(m_{\max }\right)$ of $2 \mathrm{t}$, Eq. 4:

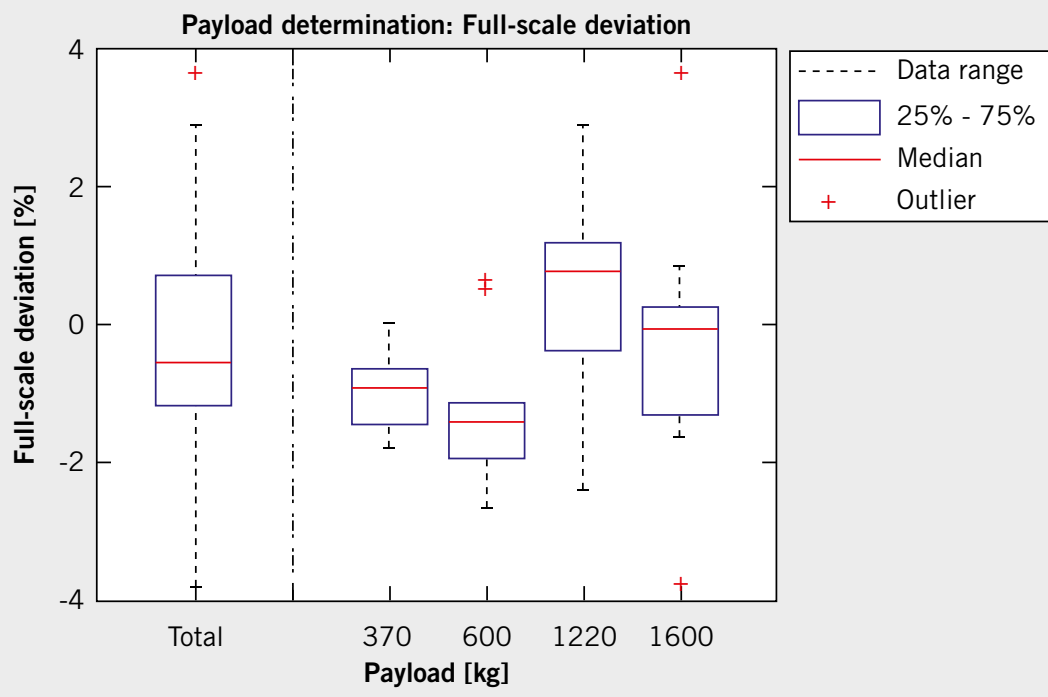




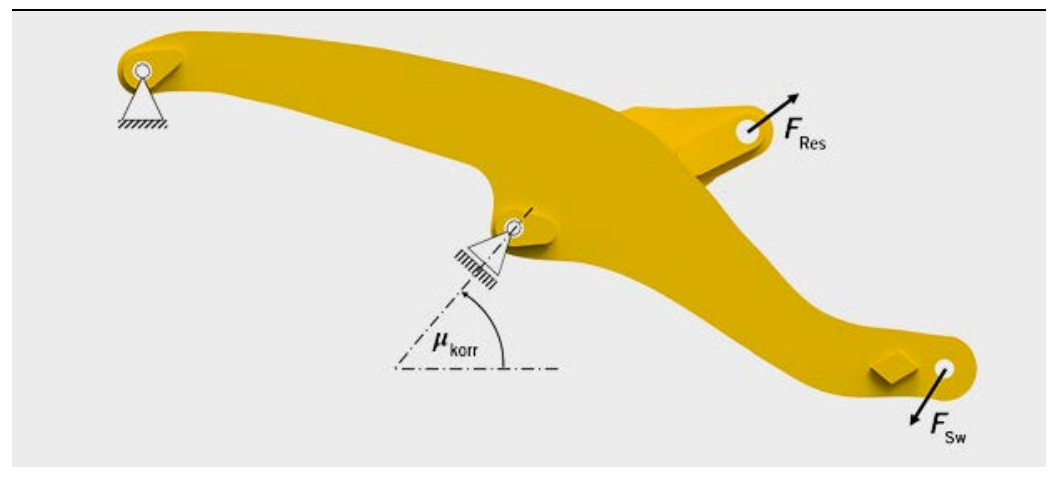

FIGURE 7 Forces acting on boom for FEM load case (C KIT)

$$
\text { Eq. } 4 \quad e_{m, F S}=\frac{\left(m_{\text {calc }}-m_{\text {real }}\right)}{m_{\max }}
$$

The red line in FIGURE 6 characterizes the median value. This corresponds to the limit at which precisely $50 \%$ of all values are larger and $50 \%$ are smaller. In the area of the box - that is between the lower and the upper quartiles - the mean $50 \%$ of deviations can be found. The length of the whiskers (top, bottom) is limited to a maximum of 1.5 times the interquartile distance and ends at the last data point within the limits. All points outside the whiskers are outliers and marked red. It can be seen that, independent of the payload, the median value differs from the real measured value by a maximum of $1.5 \% .50 \%$ of the measured values per series are within a tolerance bandwidth of maximum $2 \%$. In general, it can be seen that the payload tends to be underestimated. No trend depending on mass can be perceived. The evaluation has shown that the largest deviations occur at the maximum and minimum extension strokes of the cylinders. Considering error tolerance and reproducibility of the sensors used, the results of determining the payload are convincing and sufficiently accurate for the application.

Besides using payload determination to predict the component loads, a benefit arises with respect to other problems in the field of mechanical engineering, such as assessing process efficiency or planning construction site logistics.
The approach referred to applies only to steady-state operating points, i.e. to operating points without any movement of the kinematics of the lifting structure. To extend the approach to dynamic operating points, accelerations and inertia of masses must be taken into account as well. Moreover, for an unknown mass force, it is not only the horizontal but also the vertical lever arm which is relevant. For bulk material, the shape of the unknown mass can be approximated to a trapezoid shape by means of the shape of the bucket. This simplification results in a mass inertia and a defined position of the center of mass depending on only one variable and thus able to be solved by way of the steady-state system described above. Inclination of the machine must be taken into account in the calculation in addition.

After determining the payload, a conclusion can be drawn as to the forces in the bearing positions not taken into account as yet connection of the boom with the wheel loader and connection of the boom to the bell crank. In this way, all forces acting on the boom are known and can be used to determine a damage model in Step 3 (Part 5).

\section{STRESS CALCULATION}

For the steady-state case, the load acting on the boom can be approximated by the model shown in FIGURE 7 . Besides the forces shown, also the dead weight of the component acts upon the

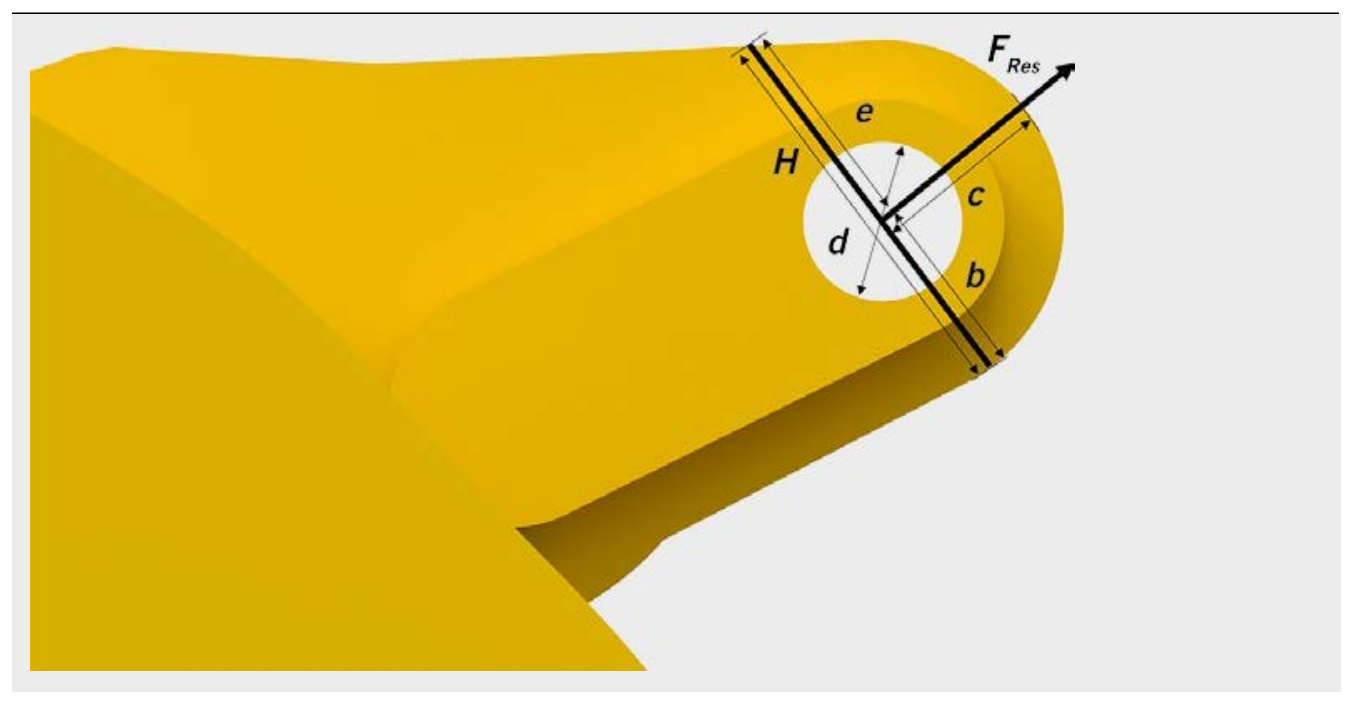


boom. For dynamic determination, the acceleration of the boom and the resulting varying amounts of forces at the bearing positions must be taken into account additionally.

To determine the most highly loaded points, a full factorial FEM study is carried out. In this step, the extension lengths of the lifting and tilting cylinders and, in addition, the payload, are varied. The digging process is not part of the current analysis. The highest stresses occasionally occur at the joints between the boom and the machine (position of the fixed bearing) and at the connecting points between the boom and the force deflector (position of force $F_{\text {Res }}$ ). The boom is modeled as one complete component; welds are not taken into account.

This information serves to develop a load model mainly databased [3] or motivated by physics. The approach presented for the connecting points is based on a physical notch model. The load related to fatigue at the points of the highest stresses of the two bearings is assumed to be the normal tensile stress with a superimposed notch effect, as the von Mises reference stress is nearly identical to the normal stress in the direction of the external force.

Under this assumption, first a nominal stress $\sigma_{\text {nom }}$ is calculated which results from the force applied to the bearing and the area normal to it. The notch case shown in the example is a mix of an eccentric bore in a plate and a joint notch according to [4]. The notch factors are calculated based on two different notch theories.

There are analytical solutions to these notch cases, which depend only on geometric relation factors [4]. For the theory of asymmetrical bore, the factors $f=\frac{e}{b}$ and $a=\frac{d}{b}$ are used according to Peterson [4]. The theory of the joint notch uses the factors $\frac{C}{H}$ and $\frac{d}{H}$. In this case, $H$ indicates the width of the plate, $c$ is the distance between the center of the bore and the outside of the component in the direction of force, and $d$ is the diameter of the bore.

The notch factor $K_{t}$ is a sub-function of all factors of the two notch cases mentioned, Eq. 5.
Eq. $5 K_{t}=k_{0}+k_{1} \cdot f+k_{2} \cdot a+k_{3} \cdot \frac{d}{H}+k_{4} \cdot \frac{C}{H}$

Eq. 6 shows a function indicating a maximum stress $\sigma_{\max }$ at the point of bearing on the basis of a common notch stress factor and the nominal stress $\sigma_{\text {nom }}$. To determine the subfunction parameter, a linear regression is carried out on the basis of FEM solutions.

$$
\text { Eq. } 6 \sigma_{\max }=K_{t}\left(f, a, \frac{d}{H}, \frac{c}{H}\right) \cdot \sigma_{\text {nom }}
$$

For parameterization of the function, a fully factorial FEM solution space is generated again. The accuracy of the FEM solution obtained has a significant impact on the quality of the notch model. The accuracy of the results typically increases with the number of nodes, which frequently results in a higher stress at the point of consideration. FIGURE $\mathbf{9}$ shows this for the example of a bearing eye for a defined load case (black curve). An increase in the number of nodes at the same time increases computation time (red curve). An increase in the computation time by a factor of 100 in this case achieves an increase in accuracy and stress of $17 \%$. According to the underlying linear damage accumulation according to Miner [5] and the FKM guideline [6], the present loading case of an unwelded component is determined by a difference in the tolerable number of load cycles $N$ and a lifetime of more than $140 \%$ (blue), Eq. 7.

$$
\text { Eq. } 7\left(\frac{\sigma_{\max , 1}}{\sigma_{\max , 2}}\right)^{5}=\frac{N_{1}}{N_{2}}
$$

As a point of reference of these considerations, the finest mesh presented with more than $12 \times 10^{6}$ nodes is chosen. The nominal stress and all geometry factors can be determined at any operat-

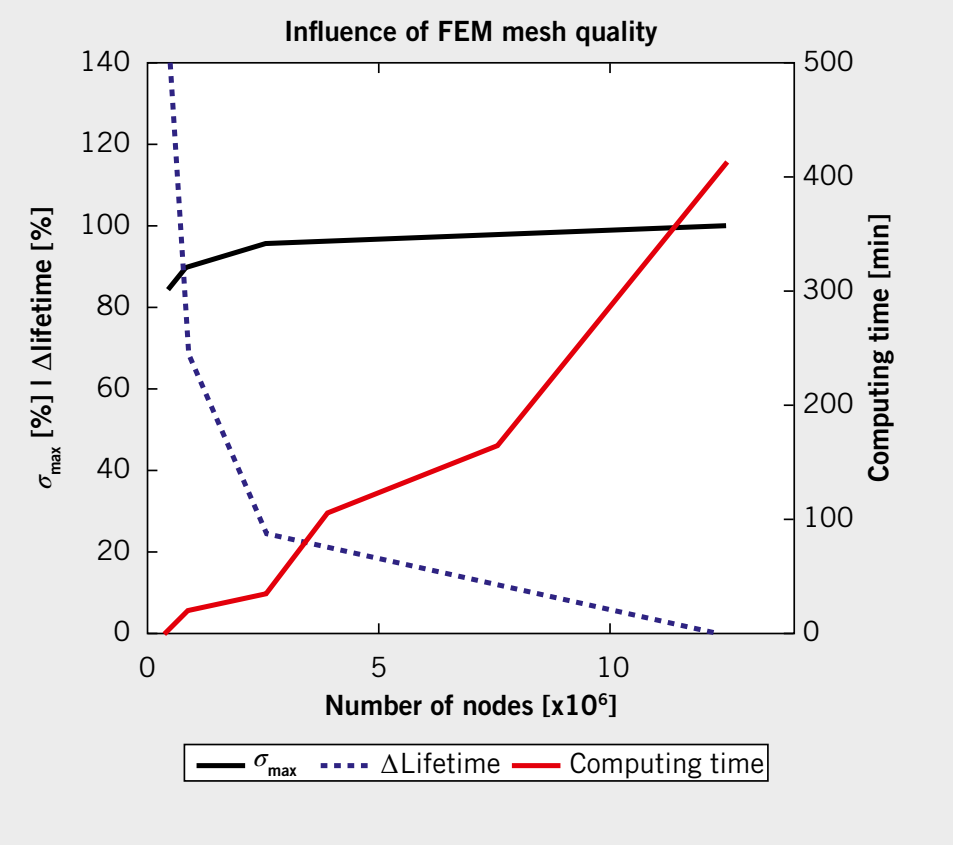

FIGURE 9 Influence of FEM mesh quality on accuracy and computation time (๑ KIT) 


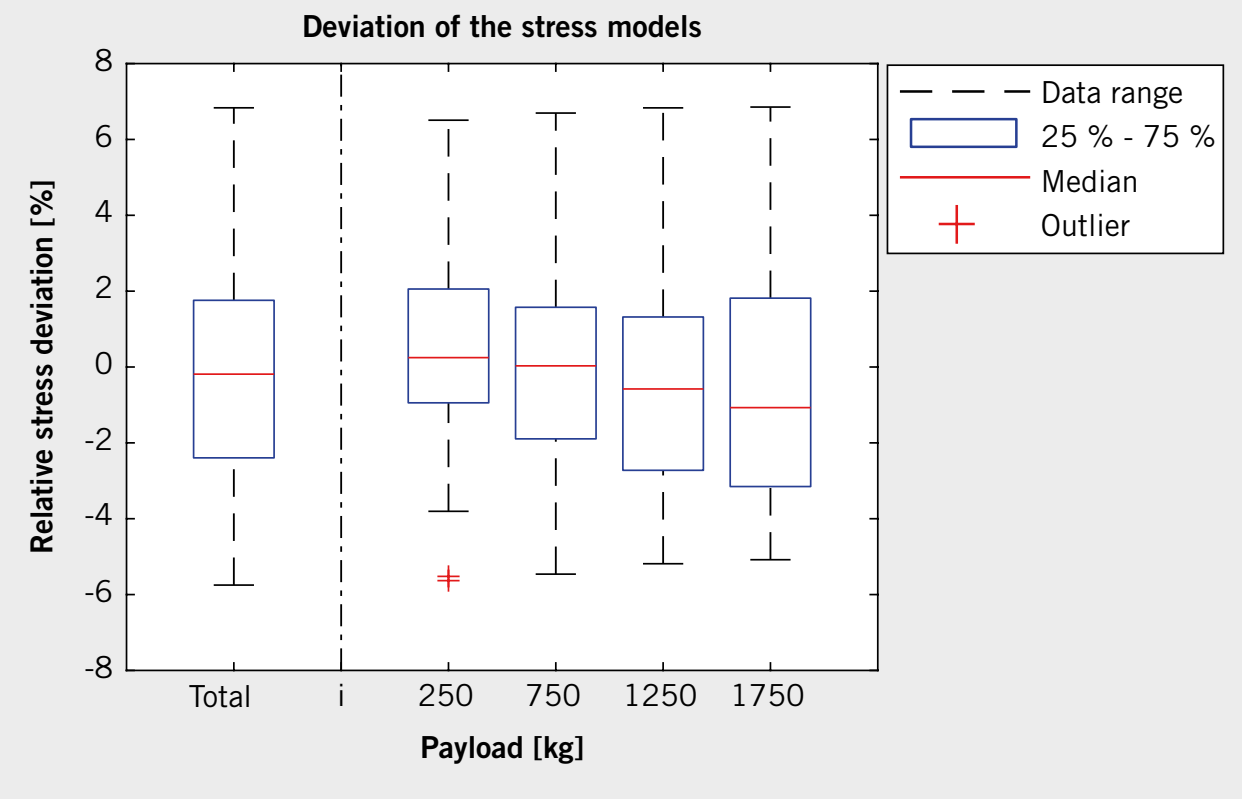

FIGURE 10 Model quality of the simplified loading models (C KIT)

ing point of the fully factorial FEM solution. This dataset is used for linear regression of the notch function. A model of the boom with $2.5 \times 10^{6}$ nodes is used to evaluate the theory.

FIGURE 10 shows a box plot of the relative stress deviation for the comparison of the two model types using the bearing eye as an example. Four different payloads with varying cylinder extension strokes are investigated for the load cases. These were not used for parameterization. For comparison, the relative stress deviation $e_{\sigma}$, was defined by the difference of the maximum stress values of the two approaches $\left(\sigma_{\mathrm{FEM}}-\sigma_{\text {lin }}\right)$ divided by the stress value of the FEM calculation $\left(\sigma_{\mathrm{FEM}}\right)$ :

$$
\text { Eq. } 8 e_{\sigma}=\frac{\sigma_{\mathrm{FEM}}-\sigma_{\text {lin }}}{\sigma_{\mathrm{FEM}}}
$$

The model comparison produces results for the simplified model, the absolute median value of the individual simulation series being less than $1 \%$. Increasing the payload reveals a trend towards higher tolerance bands, $50 \%$ of the measured values are in a tolerance band of $\pm 3 \%$. The computation time for the simplified model is several powers of 10 lower than the FEM calculation.

\section{SUMMARY AND CONCLUSION}

The method of real-time load determination described generates added value in a multitude of applications, the presented application of the wheel loader boom is only an example. Thus, any damagecritical components of the most mobile machines can be examined by such an approach.

The method presented in this contribution comprises three parts. In step 1, fundamental kinematic relations are determined.
In step 2, an estimate of the payload is carried out based on cylinder strokes and pressures. The accuracy obtained is $2 \%$ full scale, representing an excellent result for the measuring systems used. Knowing the bulk mass allows all external and dynamic forces in the subsystem of working kinematics to be calculated. Models motivated by the theory of notches and parametrized by FEM results serve to determine component loads. The accuracy of the models mainly depends on the quality of the parametrization data. In this case, an agreement of maximum stresses of $\pm 20 \mathrm{MPa}$ was achieved. The use of this method on control systems of mobile machines is already conceivable today.

This contribution describes the approach merely for steady-state and quasi-steady operating conditions. Extension by the dynamic effects occurring in real operation, in addition to strain gage based experimental validation of stress calculation, is the subject of current research work.

\section{REFERENCES}

[1] Achelpohl, E. R.: The Effect of Overloading on Reliability of Wheel Loader Structural Components. Online: https://scholarsmine.mst.edu/cgi/viewcontent. cgi? referer=https://www.google.com/\&httpsredir=1\&article=3660\&context= doctoral_dissertations, access: July 26, 2019

[2] Conn, A. R.; Gould, N. I. M.; Toint, P. L.: Trust Region Methods. Philadelphia: Society for Industrial and Applied Mathematics, 2000

[3] Brinkschulte, L.; Geimer, M.: Real-time Estimation of the Remaining Lifetime of Components, ATZoffhighway worldwide 03/2017, pp. 54-60 [4] Peterson, R. E.; Pilkey, W. D.: Peterson's stress concentration factors. Norwich: Knovel, 2003

[5] Haibach, E.: Betriebsfestigkeit - Verfahren und Daten zur Bauteilberechnung. Berlin: Springer Verlag, 2006

[6] Rennert, R.; et al.: Rechnerischer Festigkeitsnachweis für Maschinenbauteile aus Stahl, Eisenguss- und Aluminiumwerkstoffen. Frankfurt am Main: VDMAVerlag, 2012 
Karlsruher Institut für Technologie

\section{Repository KITopen}

Dies ist ein Postprint/begutachtetes Manuskript.

Empfohlene Zitierung:

Brinkschulte, L.; Hafner, J.; Geimer, M.

Real-time Load Determination of Wheel Loader Components.

2019. ATZ heavyduty worldwide, 12

doi:10.5445//R/1000099720

Zitierung der Originalveröffentlichung:

Brinkschulte, L.; Hafner, J.; Geimer, M.

Real-time Load Determination of Wheel Loader Components. 2019. ATZ heavyduty worldwide, 12 (4), 62-68.

doi:10.1007/s41321-019-0043-5 